\title{
Structural and functional analysis of the Entamoeba histolytica EhrabB gene promoter Mónica Romero-Díaz ${ }^{1}$, Consuelo Gómez ${ }^{2}$, Israel López-Reyes ${ }^{1}$, Máximo B Martínez ${ }^{3}$, Esther Orozco ${ }^{1}$ and Mario A Rodríguez*1
}

Address: ${ }^{1}$ Departamento de Patología Experimental. Centro de Investigación y de Estudios Avanzados del IPN. A.P. 14-740 México, DF 07360, México, 2 Programa Institucional de Biomedicina Molecular, ENMyH-IPN, Guillermo Massieu Helguera, No. 239. Fracc. La Escalera, Ticomán, CP 07320 México, DF, México and 3Posgrado en Ciencias Genómicas, Universidad Autónoma de la Ciudad de México, San Lorenzo \# 290, Col. Del Valle, CP 03100, México DF, México

Email: Mónica Romero-Díaz - rdmoni@yahoo.com.mx; Consuelo Gómez - cgomez@ipn.mx; Israel López-Reyes - isralo69@yahoo.com.mx; Máximo B Martínez - mmartinezb2002@yahoo.com; Esther Orozco - esther@cinvestav.mx; Mario A Rodríguez* - marodri@cinvestav.mx

* Corresponding author

Published: 20 September 2007

BMC Molecular Biology 2007, 8:82 doi:10.1/86/1471-2199-8-82
Received: 17 November 2006

Accepted: 20 September 2007

This article is available from: http://www.biomedcentral.com/147I-2199/8/82

(c) 2007 Romero-Díaz et al; licensee BioMed Central Ltd.

This is an Open Access article distributed under the terms of the Creative Commons Attribution License (http://creativecommons.org/licenses/by/2.0), which permits unrestricted use, distribution, and reproduction in any medium, provided the original work is properly cited.

\begin{abstract}
Background: The Entamoeba histolytica EhrabB gene encodes for a Rab GTPase involved in phagocytosis. It is located at a virulence locus where the Ehcp / I2 gene is in the complementary strand at 332 bp of EhrabB start codon, suggesting a finely regulated transcription of both genes. However, the transcription regulation in this parasite is poorly understood.

Results: To initiate the knowledge of EhrabB gene expression regulation, here we studied the structural characteristics of its gene promoter and its control transcription elements. In silico searches of the EhrabB 5'-flanking region revealed that it contains a motif similar to the upstream regulatory element I (UREI) of the $E$. histolytica $h g / 5$ gene. It also has sequences with homology to C/EBP and GATAI binding sites, and heat shock elements (HSE). Primer extension experiments revealed that EhrabB has at least four transcription initiation sites. The elements at the 5'-flanking region that drive EhrabB gene expression were detected and characterized using transitory transfected trophozoites with a plasmid carrying the CAT reporter gene. EhrabB transcription is negatively regulated by a sequence located between positions -49 I to -428 with respect to the first transcription initiation site. We also showed that the UREI-like motif activates EhrabB transcription. In addition, heat shock activated the EhrabB promoter in episomal constructs and lead to an increase in de novo EhrabB transcription.
\end{abstract}

Conclusion: The data suggest that EhrabB transcription is controlled negatively by an unidentified sequence, but it is activated by an UREI-like motif. Our analyses also revealed the presence of activator HSE that function under stress.

\section{Background}

Entamoeba histolytica is the protozoon responsible for up to 100,000 deaths each year [1]. Only $10 \%$ of infected people present disease symptoms expressed in intestinal or extraintestinal amoebiasis. The molecular mechanisms participating in the parasite invasiveness are not completely understood. Diverse populations of E. histolytica, including clones derived from a particular strain, display 
different virulence phenotype [2-4]. In addition, longtime cultured trophozoites, which show poor capacity to produce liver abscesses in experimental animals, recover their virulence after incubation with cholesterol or with certain types of bacteria, or after their passage by hamster livers [5-8]. This behavior could be due to changes in expression of certain genes. In fact, trophozoites isolated from infected animals present changes in gene expression with respect to trophozoites cultured in the laboratory $[9,10]$. It has been also shown that trophozoite interaction with human collagen type I and $\mathrm{Ca}^{2+}$ triggers transcription of virulence related molecules as the amoebapore $\mathrm{C}$, the cysteine proteinase 5 and a cdc48-like protein $[11,12]$.

In contrast to other eukaryotes, transcription of E. histolytica protein-coding genes is insensitive to high concentrations of alpha-amanitin [13]. In addition, certain promoters of protein-coding genes present three unusual motifs in their 5'-flanking region [14]: (a) the GTATTTAAA(G/C) sequence, which corresponds to the TATA box; (b) the AAAGAACT sequence, named GAAC element; and (c) the initiator AAAATTCA sequence (Inr). Furthermore, five upstream regulatory elements (URE1 to URE5) were identified in the promoter of the galactose-inhibitable lectin heavy subunit gene hgl5 [14]. Additionally, the structural and functional characterization of promoters driving the expression of EhPgp1 and EhPgp5 genes, whose products are implicated in the multidrug-resistance event, revealed that they contain motifs with homology to cisregulatory elements of eukaryotic cells [15-19].

Vesicular trafficking plays an important role in the pathogenic mechanism of E. histolytica, because it participates in secretion of virulence factors and in the internalization of host cells. In eukaryotes, vesicular trafficking is regulated by Rab GTPases, which by cycling between active (GTPbound) and inactive (GDP-bound) conformations act as molecular switches that integrate several events at each step of vesicular transport [20]. Thus, Rab proteins may be involved in the E. histolytica pathogenicity. EhRabB is a Rab GTPase located in small vesicles that in wild-type trophozoites are translocated to plasma membrane and to phagocytic mouths during phagocytosis [21], whereas, in trophozoites deficient in phagocytosis most of these vesicles remain in the cytoplasm [22]. In addition, Marion et al. [23], using a proteomic approach, described that EhRabB is among the proteins identified during early phagocytosis. Although EhRabB was not identified in independent phagosome protomics studies using carboxylated latex beads $[24,25]$, those results suggest that EhRabB participates in phagocytosis, an event related to the E. histolytica pathogenicity [26].
The EhrabB gene is located close to the Ehcp112 and Ehadh112 genes, whose products form the EhCPADH complex, also involved in the parasite pathogenic mechanism [27]. These three genes spans a 4500 bp region named virulence locus (VI) [28], providing a good model to study gene transcription regulation of virulence-related genes. The EhrabB gene is situated 332 bp upstream of the Ehcp112 gene, but in the complementary strand [21]. Some of the cis-elements that control the Ehadh112 and Ehcp112 transcription are located inside the open reading frame of Ehcp112 and EhrabB, respectively [28,29]. However, the cis-acting sequences which might be relevant to drive the $E h r a b B$ gene expression have not been identified yet.

To elucidate the molecular mechanisms controlling EhrabB gene expression, we studied here the structural and functional characteristics of its promoter. Our analyses revealed that it has several transcription initiation sites. We also detected the presence of one region that represses and one motif that activates EhrabB transcription. Interestingly, the gene promoter has regulatory heat shock elements that under heat shock stress activate EhrabB transcription.

\section{Results and discussion Structural analysis of the 5'-flanking region of EhrabB}

The proximity of EhrabB and Ehcp112 genes and their opposite transcription, suggest a finely regulated gene expression of these two genes. To initiate the comprehension of the mechanisms involved in their transcription, we studied here the EhrabB promoter region, comprising from the start codon to -755 bp upstream of this site. In this region we looked for: (a) consensus sequences described in E. histolytica core promoters [14]; (b) sequences with similarity to the URE1 to URE5 elements [14]; and (c) consensus sequences described for binding of eukaryotic transcription factors.

Neither E. histolytica Inr elements nor TATA-box sequences were identified in the EhrabB promoter region. In contrast, we detected a sequence similar to the GAAC element at 22 to 29 nucleotides upstream of the start codon (Fig. 1). The absence of Inr and TATA box consensus sequences suggests that there is a particular unknown transcription initiation mechanism for EhrabB. Our search also revealed a sequence located at -423 to -413 that is $81.8 \%$ identical to the URE1 sequence described as a transcriptional activator of the hgl5 gene [14] (Fig. 1). The analysis also showed the presence of three C/EBP, one GATA-1 and seven heat shock elements (HSE) consensus sequences in this region (Fig. 1). 


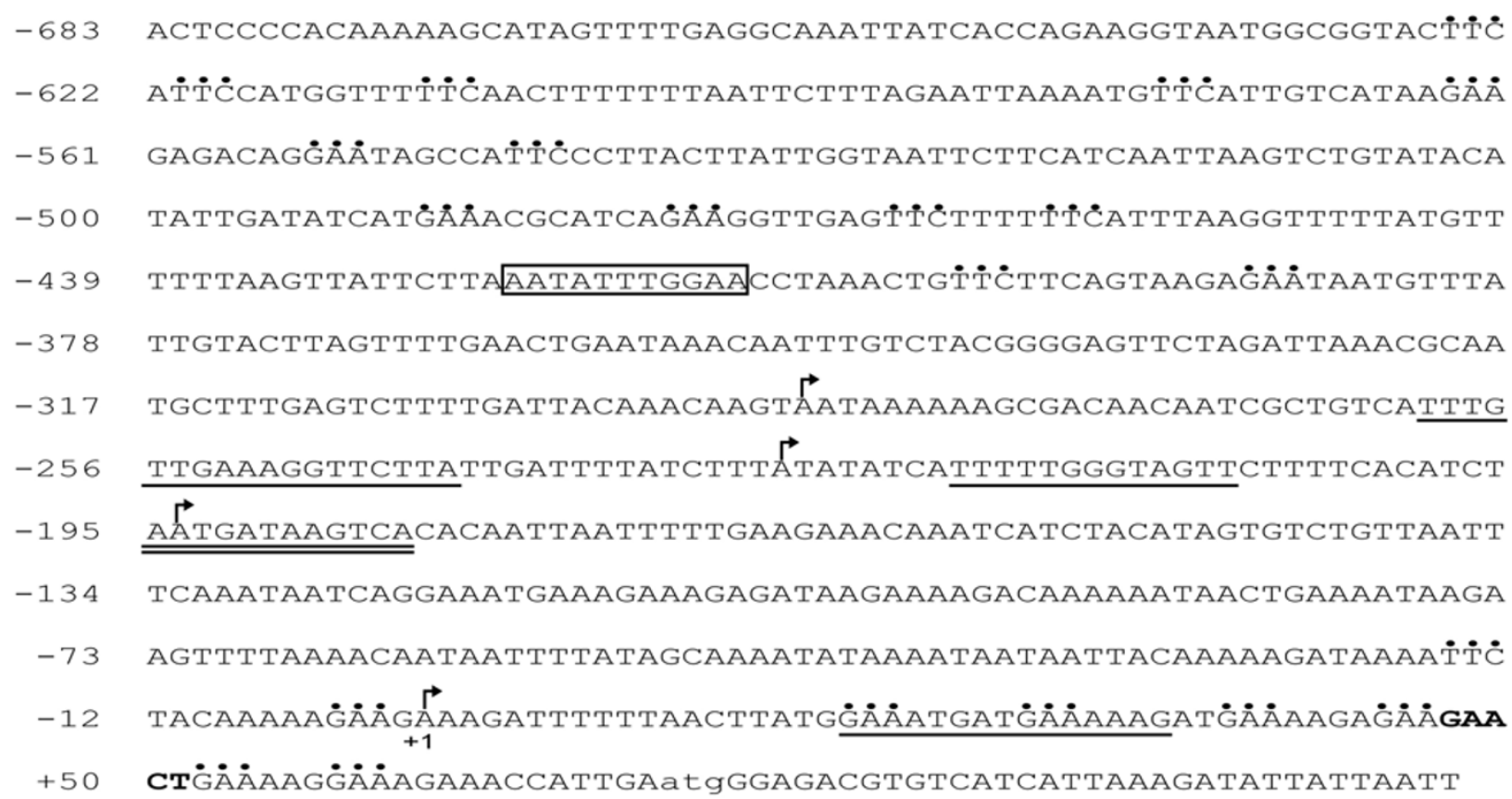

\section{Figure I}

Primary structure of the EhrabB promoter gene. Nucleotide sequence of the 755 bp upstream from the ATG (lower case). The transcription initiation sites are marked by arrows. UREI-like sequence is boxed. Putative HSE sequences are shown by dots. Putative C/EBP sequences are underlined. Putative GATA-I sequence is double underlined. Putative GAAC sequence is in bold. Numbering of nucleotides is from $+I$ at the first transcription start site.

\section{Determination of the EhrabB transcription start site}

The EhrabB gene transcription initiation site was mapped by primer extension experiments using a [ $\left.{ }^{32} \mathrm{P}\right]$-labeled 18 bp antisense oligonucleotide that spans from 85 to 68 nucleotides inside the gene open reading frame. As often found in TATA-less promoters [30], EhrabB gene exhibited several transcription initiation sites located at -72, -266, 300 and -360 , with respect to its start codon (Fig. 2A). The adenine residue located at the start of the shortest EhrabB transcript was arbitrarily designed as position +1 (Fig. 1). The AAAAGAAGA $_{+1}$ sequence, where this transcript starts, differs from the consensus sequence described for the transcription initiation site $\left(\right.$ AAAATTCA $_{+1}$ ) of the majority of E. histolytica genes [14]. On the other hand, the GAAC element, a C/EBP site and two HSE sequences found by in silico searches appeared situated downstream of the first transcription initiation site (Fig. 1), but we do not know yet their relevance in transcriptional activity.

Most of the E. histolytica genes examined so far have a short (0-21 bp) 5' untranslated region (5'UTR) [31]. EhrabB as other few E. histolytica transcripts [27,32,33] have long 5'UTR, which could have a specific role in translation regulation [34]. The shortest 5'UTR of EhrabB is composed of 72 nucleotides, whereas the longest has 360 nucleotides and the Ehcp112 transcript, which runs in opposite direction, has a 280 nucleotides 5'UTR [27]. On the other hand, the EhrabB start codon is located at $332 \mathrm{bp}$ upstream of the Ehcp112 start codon, thus, both transcripts overlap in at least 20 nucleotides (Fig. 2B). This act supports the hypothesis that expression of EhrabB and Ehcp112 genes are co-regulated.

\section{Delimitation and characterization of the EhrabB gene promoter and its relevant motifs}

The active regions of EhrabB gene promoter were characterized in trophozoites transfected with the pBSCAT-ACT vector [15] in which we cloned DNA fragments containing 96 bp downstream of the first transcription initiation site and distinct lengths fragments of the upstream region. The larger fragment (-683 to +96 ; pRab683), containing the GATA-1 ( -195 to -184$), \mathrm{C} / \mathrm{EBP}(-260$ to -243 and -220 to -208$)$, URE1 (-423 to -413$)$, and HSE (-624 to -608 , 577 to $-543,-488$ to $-458,-403$ to -388 , and -15 to -2 ) sequences, showed only $15 \pm 1 \%$ CAT activity of the one exhibited by actin gene promoter [35], used here as a control (Fig. 3B). Interestingly, in pRab428 plasmid (-428 to $+96)$, where three out of five HSE motifs present were 
A

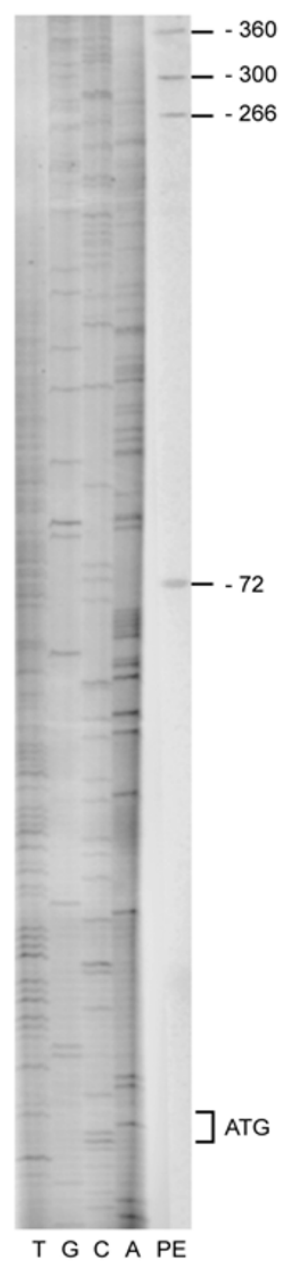

B

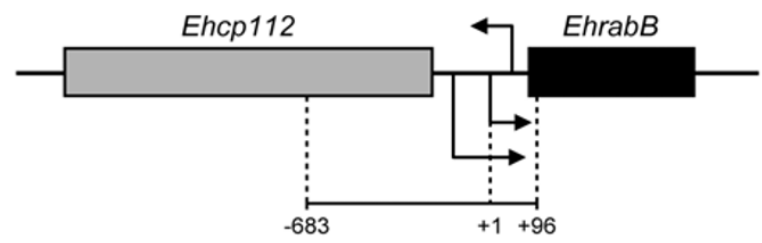

Figure 2

Transcriptional start sites of the EhrabB gene. (A) Primer extension products $(\mathrm{PE})$ were analyzed alongside sequencing ladder extended with the same 18 bp primer complementary to nucleotides 85-68 downstream of the EhrabB start codon. ATG indicate the start codon. (B) Schematic representation of the genomic organization of Ehcp / / 2 and EhrabB genes. Arrows indicate the transcription initiation sites and transcription sense for each gene. The line below shows the EhrabB 5'-flanking region analyzed (+96 to -683 ). deleted, CAT activity augmented in $41 \%$ with respect to pRab683, being $56 \pm 17 \%$ of the actin gene promoter (Fig. 3B). The construction pRab257 (-257 to +96$)$, where the URE1 ( -423 to -413$)$ and other HSE (-403 to -388$)$ were eliminated, showed $23 \pm 6 \%$ CAT activity with respect to activity displayed by the actin promoter (Fig. 3B), whereas pRab147 (-147 to +96) and pRab39 (-39 to +96) constructions, displayed $10 \pm 5 \%$ and $14 \pm 4 \%$ CAT activity, respectively (Fig. 3B). These results showed that: i) the C/ EBP (-260 to -243 and -220 to -208$)$ and GATA-1 (-195 to -184) sequences may not be relevant for EhrabB gene transcription, because their removal did not show significant effect in CAT activity; ii) a DNA region located between positions -428 to -683 negatively controls EhrabB transcription; and iii) a DNA fragment located at -257 to -428 , where a HSE and a URE1 motifs were detected, activates EhrabB transcription.

Due to the distance between EhrabB and Ehcp112 genes and to their transcription in opposite directions (Fig. 2B) is probable that our constructs could display transcription from the opposite strand to the reporter gene, producing an antisense RNA that possibly affect the promoter assays, and differences in CAT activity between pRab428 and pRab683 could be due to variations in the antisense level. To investigate if antisense RNA of the cat reporter gene is transcribed in the transfected trophozoites, we carried out strand-specific RT-PCR assays. In both constructs (pRab683 and pRab428), RT-PCR assays produced the expected fragment when we used oligo(dT) or antisense primers to synthesize cDNA, whereas no amplification was obtained when a sense primer was utilized (Fig. 3C), indicating that antisense RNA is not produced in the transfectants. These results revealed that transcriptional differences observed in transfected trophozoites are due to promoter activities, and they are according with those obtained on the transcriptional analysis of the Ehcp112 gene [28], which demonstrated that the 332 bp intergenic region between Ehcp112 and EhrabB is not able to promote the Ehcp112 transcription, and that sequences driving the Ehcp112 transcription are located inside the coding region of EhrabB (between 681 and 833 bp upstream of the Ehcp112 start codon). In our constructs this region was replaced by the cat reporter gene, consequently the sequences driving the transcription of antisense RNA are absent.

\section{Delimitation of the repressor region in EhrabB gene promoter}

According with our analyses, in the DNA fragment that inhibits EhrabB transcription (-428 to -683) we did not identify consensus transcription repressor sequences described in other systems. Thus, to delimitate the cis-elements that negatively control EhrabB transcription, we 
A

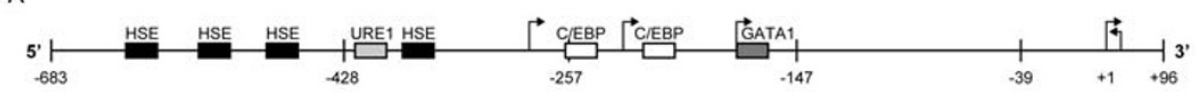

B

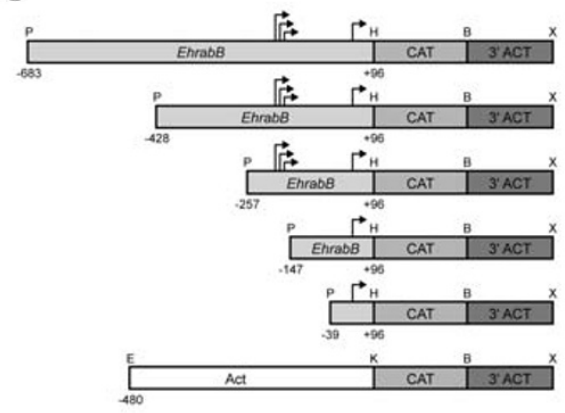

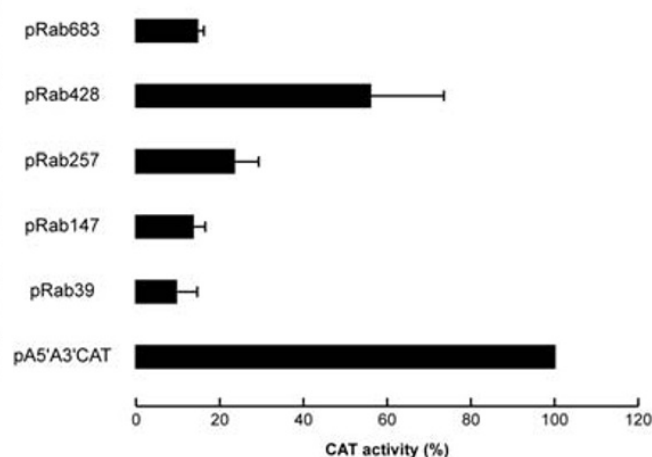

C

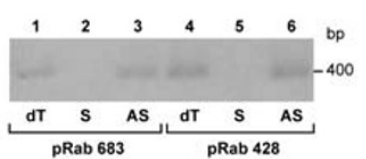

Figure 3

Promoter activity of different fragments from the 5'-flanking region of the EhrabB gene. (A) Schematic representation of the EhrabB promoter region and its putative cis-acting consensus sequences (boxes). Arrows indicate the transcription initiation sites. Arrow with sense to left show the transcription initiation site and transcription sense of the Ehcp / / 2 gene. (B) left, schematic representation of the relevant features of the plasmids used for promoter activity assays. EhrabB, 5'-flanking fragments of EhrabB, containing different 5 '-ends (from -683 to -39 upstream of the first initiation transcription site) and 96 nt downstream from the first initiation transcription site (+96). CAT, chloramphenicol acetyl transferase reporter gene. 3'ACT, 3'-flanking region of the actin gene. Act, 480 bp of the 5'-flanking region of actin gene. P, Pstl. H, HindllI. B, BamHI. X, Xhol. E, EcoRI. K, Kpnl. Arrows indicate the transcription initiation sites. Right, CAT activities obtained from trophozoites transfected with constructions at left. Activities are relative to that obtained with the actin promoter gene, used as positive control. Each bar corresponds to the average of CAT activities \pm S.D. representative of three independent experiments performed by duplicate. (C) Strand-specific RT-PCR of cat reporter gene. cDNA of trophozoites transfected with pRab683 (lanes I-3) or with pRab428 (lanes 4-6) was synthesized using sense (lanes 2, 5) or antisense (lanes 3,6) primers of the cat gene; then, PCR assays were performed and analyzed in agarose gels. Lanes I and 4, PCRs using CDNA synthesized with an oligo(dT) primer.

performed sequential deletions between position -683 and -428. Different length fragments were again cloned into the pBSCAT-ACT vector and transfected into trophozoites, then, CAT activities were measured (Fig. 4A,B). In construction comprising from -491 to +96 nt (pRab491) CAT activity was $50 \pm 3 \%$ of pRab428 (Fig. $4 \mathrm{~B}$ ) and the pRab555 $(-555$ to +96$)$ showed a dramatic drop of activity $(25 \pm 11 \%$ of pRab428) (Fig. 4B). These experiments indicated that the 67 bp region located between positions 491 to -428 contains transcription inhibitors. In this region we only detected a HSE element (-488 to -458$)$.

EMSA experiments using a 63 bp DNA probe containing nucleotides form -491 to -428 confirmed the presence of nuclear proteins interacting with this region. Results showed the formation of three DNA-protein complexes (Fig. 4C) that were competed by 150-fold excess of the same cold-probe, but remained in the presence of 350fold excess of the nonspecific competitor (Fig. 4C), indicating that DNA-protein interactions were specific. The complexes formation strengthened the hypothesis that this region has a functional repressor activity in EhrabB gene promoter.

\section{Delimitation of the activator region in EhrabB gene promoter}

To delimitate the cis-elements that activate the EhrabB transcription, we generated constructions with sequential deletions between position -428 and -257 that were cloned into the pBSCAT-ACT vector (Fig. 5A,B). Activity exhibited by extracts from trophozoites transfected with plasmids pRab369 $(-369$ to +96$)$, pRab321 $(-321$ to +96$)$, and pRab273 (-273 to +96$)$ decreased to 33 to $44 \%$ of activity displayed by the pRab428 plasmid (Fig. 5B). Constructions with additional different deletions did show similar CAT activity than pRab369. These experiments indicated that cis-elements that activate EhrabB gene transcription are located between positions -428 to $-369 \mathrm{nt}$. In this region we identified a sequence $(-423$ to -413$)$ with $81.8 \%$ identity to URE1 sequence described for the $h g l 5$ gene promoter [14] and one putative HSE motif (-403 to -388) (Fig. 5A). 
A

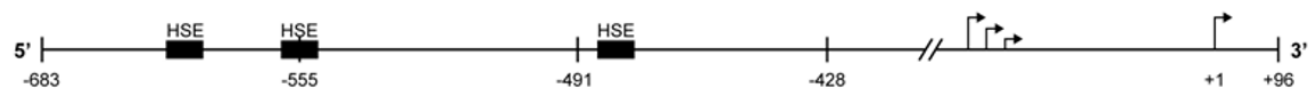

B

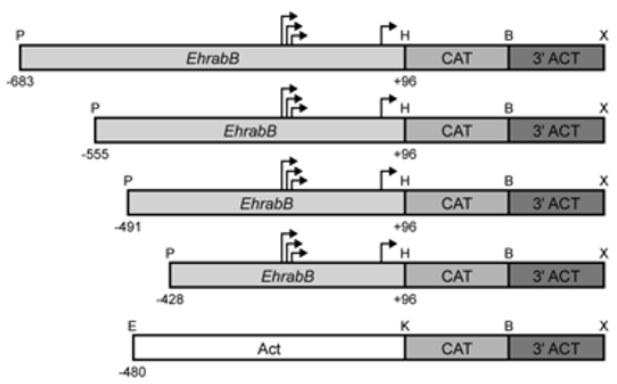

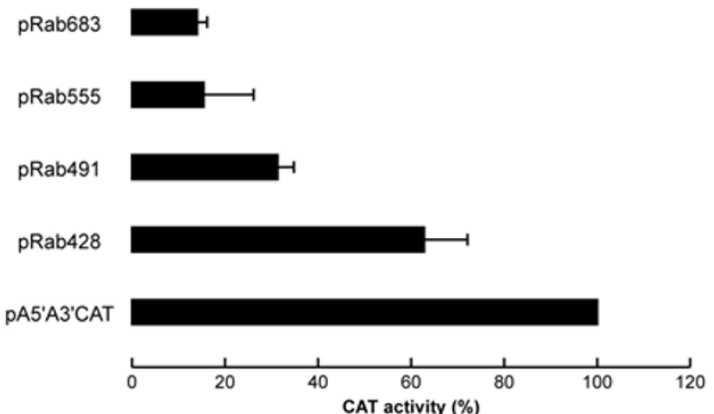

C

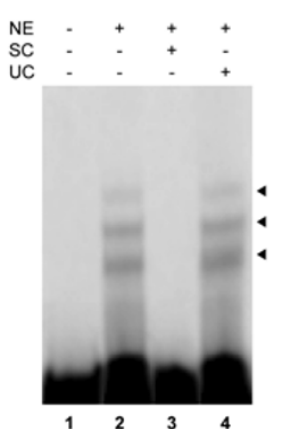

\section{Figure 4}

Delimitation of the repressive region of EhrabB transcription. (A) Schematic representation of the EhrabB promoter region analyzed and its putative cis-acting consensus sequences (boxes). Arrows indicate the transcription initiation sites. (B) Left, schematic representation of the relevant features of the plasmids used for promoter activity assays. EhrabB, 5'-flanking fragments of EhrabB containing from -683 to -428 and $+96 \mathrm{nt}$ from the first transcription initiation site (+96). Right, CAT activities obtained from trophozoites transfected with constructions at left. Activities are relative to that obtained with the actin promoter gene, used as positive control. Each bar corresponds to the average of CAT activities \pm S.D. representative of three independent experiments performed by duplicate. (C) EMSA using the ${ }^{32}$ P-labeled fragment from -49 I to -428 of EhrabB promoter as a probe and $30 \mu \mathrm{g}$ of nuclear extracts (NE) from trophozoites. Lane I, free probe. Lane 2, no competitor. Lane 3, specific competitor (SC) (I50-fold excess of the same cold fragment). Lane 4, unspecific competitor (UC) (350-fold excess of poly $[\mathrm{d}(\mathrm{I}-\mathrm{C})]$ ). Arrowheads indicate DNA-protein complexes formed by interaction of the probe with nuclear extracts.

EMSA experiments using nuclear extracts and the 59 bp DNA fragment comprising from -428 to -369 revealed that two DNA-protein complexes are formed in this region. Complexes were competed by 150 -fold excess of the same cold-sequence, but not by the presence of 350fold excess of the nonspecific competitor (Fig. 5C). Complexes formation supported the hypothesis that in this DNA fragment are situated cis-elements involved in EhrabB transcription activation.

\section{Role of the UREI-like sequence in the EhrabB transcription}

The 59 bp fragment between -428 and -369 activates transcription, forms complexes with some nuclear proteins and contains a sequence that has nine out eleven base pairs identical to the URE1 motif of $h g l 5$ (81.8\%). To analyze the role of the URE1-like sequence in EhrabB promoter activity, we deleted 13 bp containing this sequence and cloned a DNA fragment from -415 to +96 in front of the CAT reporter gene (pRab415) (Fig. 6A,B). This construct only exhibited $33 \pm 4 \%$ CAT activity of pRab428 that includes the URE1-like sequence (Fig. 6B). Our results show that the URE1-like sequence located from 423 to -413 bp is a cis-activating element of EhrabB transcription.

We also carried out EMSA experiments using the DNA fragment containing from -428 to $-412 \mathrm{bp}$ as a probe. Two DNA-protein complexes were detected in these assays (Fig. 6C). Both complexes were competed by a 150-fold excess of the same cold sequence, but they were not competed by a 150-fold excess of a nonspecific competitor with similar base composition to URE1, but mingled sequence (Fig. 6C). Interestingly, when we used as competitor a fragment containing the URE1 sequence from the hgl5 promoter, complexes were competed and they disappeared (Fig. 6C, lane 4). Moreover, two DNA-protein complexes with similar mobility were detected when this sequence was used as a probe (Fig. 6C, lane 6). These results evidenced that similar nuclear factor(s) recognized the URE1 sequence of the hgl5 gene promoter and the URE1-like sequence present in EhrabB gene promoter. 
A

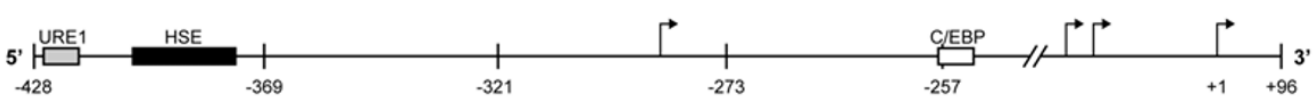

B
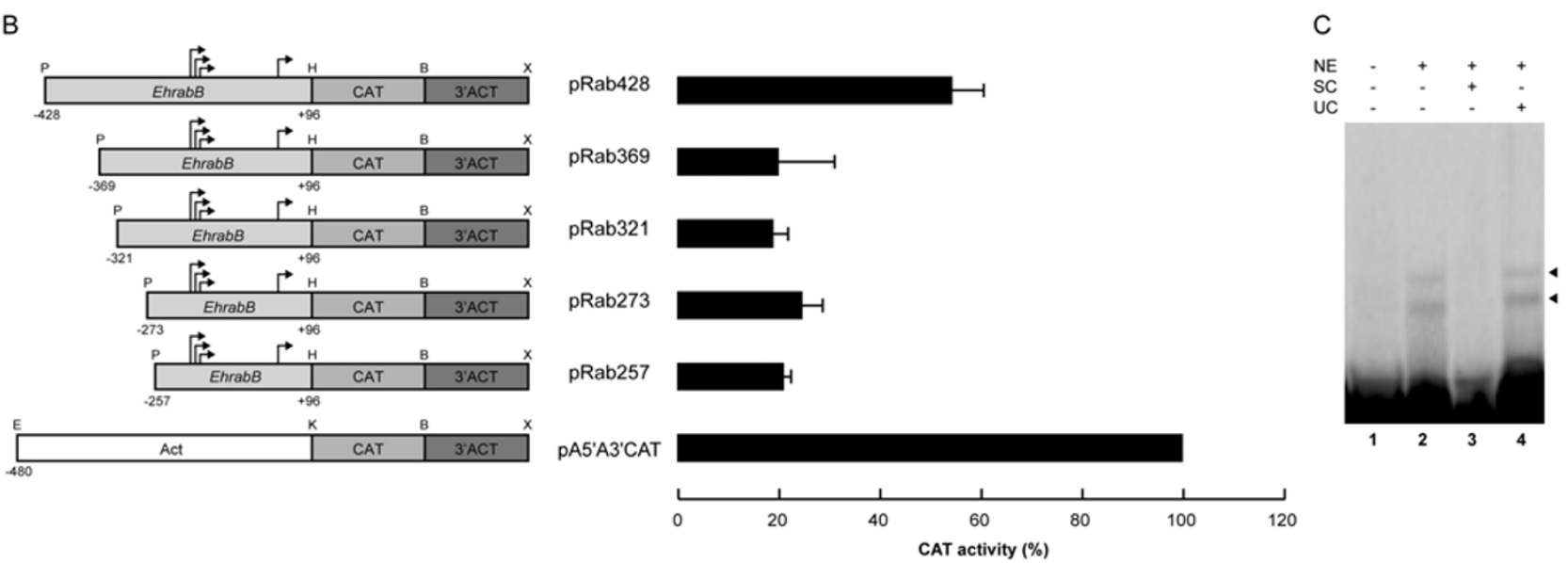

Figure 5

Delimitation of the activating region of EhrabB transcription. (A) Schematic representation of the EhrabB promoter region analyzed and its putative cis-acting consensus sequences (boxes). Arrows indicate the transcription initiation sites. (B) Left, schematic representation of the relevant features of the plasmids used for promoter activity assays. EhrabB, 5'-flanking fragments of EhrabB containing from -428 to -257 and +96 nt from the first transcription initiation site (+96).Right, CAT activities obtained from trophozoites transfected with constructions showed at left. Activities are relative to that obtained with the actin promoter gene, used as positive control. Each bar corresponds to the average of CAT activities \pm S.D. representative of three independent experiments performed by duplicate. (C) EMSA using the 32P-labeled fragment from -428 to -369 of EhrabB promoter as a probe and $30 \mu \mathrm{g}$ of nuclear extracts (NE) from trophozoites. Lane I, free probe. Lane 2, no competitor. Lane 3, specific competitor (SC) (I50-fold excess of the same cold fragment). Lane 4, unspecific competitor (UC) (350-fold excess of poly $[\mathrm{d}(\mathrm{I}-\mathrm{C})]$ ). Arrowheads indicate the two DNA-protein complexes formed by interaction of the probe with NE.

Effect of heat shock on EhrabB gene transcription

Comparative analyses of the 5'-flanking region of genes induced by heat shock have revealed that they contain tracts with three or more nGAAn or nTTCn sequences, which are essential for heat induction response [36]. These tracts are called HSE and the presence of multiple HSE can act in a cooperative way. As mentioned before, in the EhrabB 5'-flanking region we found seven putative HSEs, five of them situated upstream of the first start transcription site $(-624$ to $-608,-577$ to $-543,-488$ to -458 , 403 to -388 , and -15 to -2 ) and the two other at +20 to +39 and +44 to +60 (Fig. 1). To prove that they have a functional role in the EhrabB transcription, we used the construction pRab683 $(-683$ to +96$)$ that includes all these sequences (Fig. 7A) to transfect E. histolytica. Its ability to drive CAT expression was measured 2 and $4 \mathrm{~h}$ after trophozoites incubation at $42^{\circ} \mathrm{C}$. CAT enzymatic activity augmented approximately twice on heat shocked trophozoites with respect to the activity displayed by cells maintained at $37^{\circ} \mathrm{C}$ (Fig. 7B). Our results indicate that HSE motifs present into the EhrabB gene promoter could be functional under heat shock stress.
On the other hand, to verify the effect of heat shock in EhrabB gene transcription, we performed real-time RTPCR assays using RNA isolated from trophozoites maintained at $37^{\circ} \mathrm{C}$ or from trophozoites incubated $4 \mathrm{~h}$ at $42^{\circ} \mathrm{C}$. In concordance with CAT assays, trophozoites under heat shock presented a five-fold increase of $E h r a b B$ transcription in comparison with those grown at $37^{\circ} \mathrm{C}$ (Fig. 7C). These results support the hypothesis that under heat shock of trophozoites the HSE sequences found in the $E h r a b B$ gene promoter regulate the transcription of this gene. It is possible that these elements also regulate the gene transcription under other stimuli such as oxidative stress, during invasion, or stress induced by anti-E. histolytica drugs. However, this assumption needs to be proved.

Gene expression analysis by microarray assays revealed that heat shock treatment of E. histolytica trophozoites induce the expression of virulence-related genes such as the cysteine proteases 4 and 6, and specific alleles of the Gal/GalNac lectin [37]. In addition, some Rab GTPases are upregulated under stress conditions in other organ- 
A

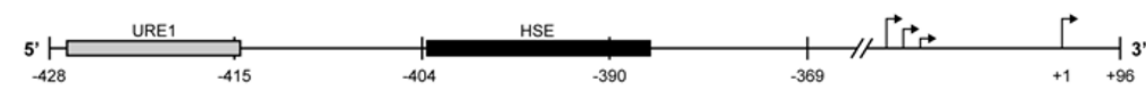

B
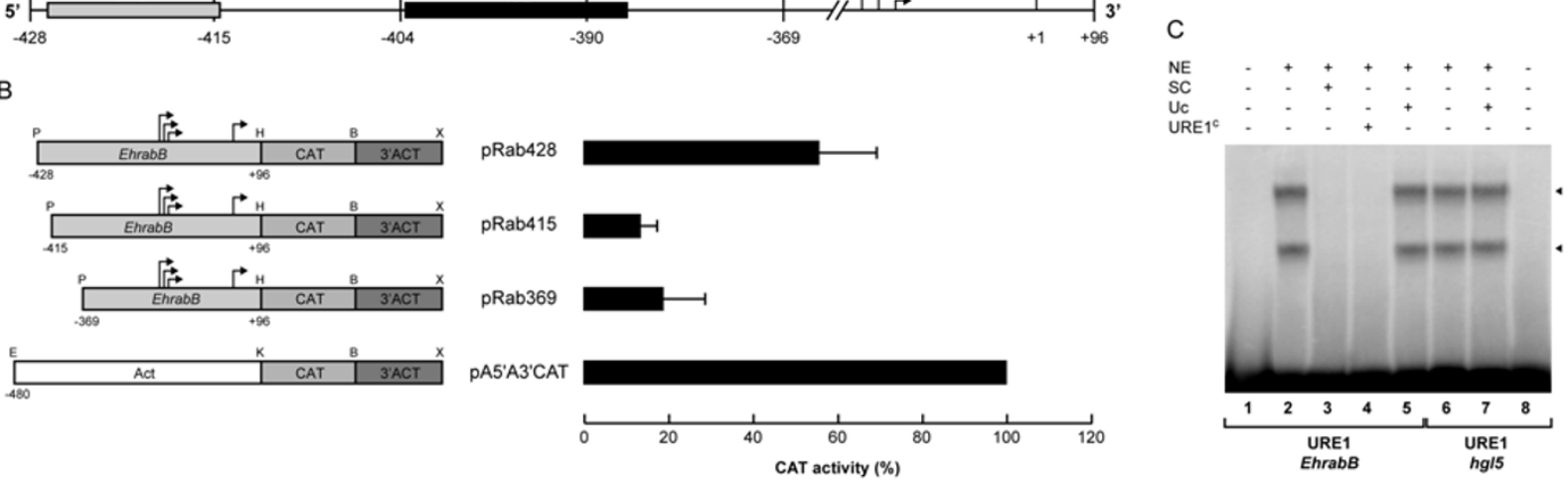

\section{Figure 6}

Role of URE I in the transcriptional activity of EhrabB. (A) Schematic representation of the EhrabB promoter region analyzed and its putative cis-acting consensus sequences (boxes). (B) Left, schematic representation of the relevant features of the plasmids used for promoter activity assays. EhrabB, 5'-flanking fragments of EhrabB, containing -428 (pRab428), -415 (pRab4 I5) (without the UREI-like sequence), and -369 (pRab369) nt from the first transcription initiation site.Right, CAT activities obtained from trophozoites transfected with constructions showed at left. Activities are relative to that obtained with actin promoter gene, used as positive control. Each bar corresponds to the average of CAT activities \pm S.D. representative of three independent experiments performed by duplicate. (C) EMSA using the 32P-labeled sequences UREI from EhrabB (UREI EhrabB) or from hgl5 (URE hgl5) gene promoters as probes and $30 \mu \mathrm{g}$ of trophozoite nuclear extracts (NE). Lanes I-5, EMSA using the UREI sequence from EhrabB as a probe. Lane I, free probe. Lane 2, no competitor. Lane 3, specific competitor (SC) (I50-fold excess of the same cold fragment). Lane 4, UREI from the hgl5 gene promoter used as competitor (UREIC) (I50-fold excess). Lane 5, unspecific competitor (UC) (I50-fold excess of a mingled oligonucleotide with similar composition). Lanes 68, EMSA using the UREI sequence from hgl5 as a probe. Lane 6, no competitor. Lane 7, unspecific competitor (UC) (I50-fold excess of a mingled oligonucleotide with similar composition). Lane 8, free probe. Arrowheads indicate the two DNA-protein complexes formed by probe-nuclear extracts interaction.

isms [38-40], indicating that these proteins may play an important role in cell adaptation to stress, beyond their housekeeping function in intracellular vesicle trafficking. In Saccharomyces cervisiae the HSE sequences are also involved in the transcription induction not only of heat shock proteins (HSP) but also of genes encoding proteins involved in diverse cellular processes [41].

\section{Conclusion}

We showed here that the EhrabB gene is transcriptionally activated by heat shock and that the EhrabB gene promoter contains cis-elements that repress and activate the gene transcription on normal conditions. The cis-activating element corresponds to a URE1-like sequence, whereas de cis-repressive element remains to be identified.

\section{Methods \\ E. histolytica cultures}

Trophozoites of clone A [26], strain HM1:IMSS, were axenically cultured in TYI-S-33 medium and harvested during logarithmic growth phase as described [42].

\section{Sequence analysis of the 5'-flanking region of EhrabB}

The 5'-flanking region of the EhrabB gene, which contains its putative promoter, was previously isolated from a genomic clone that includes the Ehadh112, Ehcp112 and EhrabB genes [GeneBank: AF172320]. The EhrabB upstream sequence is identical to the sequence found in the E. histolytica genome project [43]. Prediction of putative cis-acting elements in $E h R a b B$ gene promoter was done using the TRANSFAC database [44]. For searching cis-acting elements found in other E. histolytica genes (TATA box, GAAC element, Inr and URE sequences) we used the LALIGN program [45].

\section{Primer extension, RT-PCR and real-time RT-PCR assays}

Total E. histolytica RNA was obtained using the TRIZOL reagent (GIBCO BRL) according to the manufacturer recommendations. For primer extension assays, $40 \mu \mathrm{g}$ of RNA were hybridized to an 18 bp [ $\left.{ }^{32} \mathrm{P}\right]$-labeled primer ( $5^{\prime}-$ GGTCACAATACTGACGAA-3') complementary to nucleotides 85-68 downstream to the start codon of the EhrabB gene. Annealing was performed as previously described [15]. Nucleic acids were phenol-chloroform extracted, 


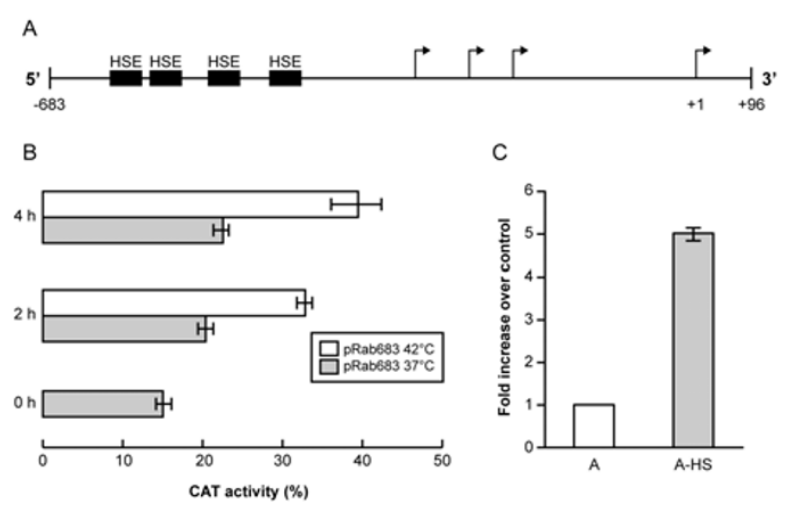

Figure 7

Effect of heat shock on EhrabB transcription. (A) Schematic representation of the EhrabB promoter region and its putative cis-acting consensus sequences (boxes). Arrows indicate the transcription initiation sites. (B) The 683 bp DNA fragment from the 5'-flanking region of EhrabB was cloned upstream of the cat reporter gene into the PBSCAT$A C T$ vector. $E$. histolytica trophozoites were transfected with this construction and incubated at $37^{\circ} \mathrm{C}$ for $48 \mathrm{~h}$. Then, trophozoites were incubated for 2 and $4 \mathrm{~h}$ more at $37^{\circ} \mathrm{C}$ or at $42^{\circ} \mathrm{C}$ and CAT activities were determined. Activities are relative to that obtained with the actin promoter gene at $37^{\circ} \mathrm{C}$, used as positive control. Each bar corresponds to the average of CAT activities \pm S.D. representative of three independent experiments performed by duplicate. (C) Real-time RT-PCR assays using RNA from trophozoites grown at $37^{\circ} \mathrm{C}$ (A) or after $4 \mathrm{~h}$ of heat shock treatment (A-HS) and specific primers for EhrabB. Amplification of the E. histolytical $8 \mathrm{~S}$ rRNA was used as normalizer.

ethanol precipitated, and separated by electrophoresis on $6 \%$ urea-polyacrylamide gels. A sequencing ladder was generated using the above oligonucleotide and the plasmid containing the 5'-flanking region of the EhrabB gene as template. Gels were visualized with a phosphoimager analyzer (BIO-RAD).

For strand-specific RT-PCR of the cat reporter gene we used specific sense (5'-ATGGAGAAAAAAATCACTGGATATA-3') or antisense (5'-ATAGGCCAGGTTTTCACCGTAACAC-3') oligonucleotides for the cDNA synthesis, and then PCR assays were performed using the same primers. Amplification conditions were as follows: $94^{\circ} \mathrm{C}$ for $5 \mathrm{~min}$; then 30 cycles at $94^{\circ} \mathrm{C}$ for $40 \mathrm{~s}, 57^{\circ} \mathrm{C}$ for $35 \mathrm{~s}$ and $72^{\circ} \mathrm{C}$ for $40 \mathrm{~s}$; and a final extension at $72^{\circ} \mathrm{C}$ for $7 \mathrm{~min}$.

To evaluate the transcription of EhrabB under heat shock by real-time RT-PCR, total RNA was isolated from trophozoites grown at $37^{\circ} \mathrm{C}$, or $4 \mathrm{~h}$ after incubation at $42^{\circ} \mathrm{C}$, and cDNA was synthesized using an oligo(dT) primer (Invitrogen). Specific primers for EhrabB (sense: 5'-GTGTCG-
GGAAGACAGCGTTAC-3'; antisense: 5'CTTGTCCTGCAGTATCCCAAAGT-3') were designed using the Primer Express software (Applied Biosystems). Amplification of the E. histolytica $18 \mathrm{~S}$ rRNA using primers Ehd-239F (5'-ATTGTCGTGGCATCCTAACTCA-3') and Ehd-88R (5'-GCGGACGGCTCATTATAACA-3') [46] was used as normalizer. Quantitative amplifications were performed in the 7300/7500/7500 Fast Real-Time PCR System (Applied Biosystems) using the SYBER Green PCR Master Mix kit (Applied Biosystems). Amplification conditions were as follows: 30 cycles at $95^{\circ} \mathrm{C}$ for $20 \mathrm{~s}, 55^{\circ} \mathrm{C}$ for $20 \mathrm{~s}$, and $72^{\circ} \mathrm{C}$ for $35 \mathrm{~s}$. Two replicates were analyzed in triplicate. Relative quantification was performed using the delta delta ct method [47].

\section{Plasmids construction}

Different regions of the EhrabB promoter were inserted into the pBSCAT-ACT plasmid in front of the CAT reporter gene followed by the 3'-flanking sequence of the E. histolytica actin gene [15]. All fragments contained $24 \mathrm{bp}$ of the 5 '-end coding region of the EhrabB gene and were generated by PCR using Pfx DNA polymerase (Invitrogen), specific primers (Table 1), and the genomic clone containing the EhrabB upstream flanking region as template. The orientation and sequence of each construct were confirmed by DNA sequencing. As a positive control we used the pA5'A3'CAT vector containing the E. histolytica actin gene promoter [35] and as negative control the reporter vector without promoter region (pBSCAT-ACT).

\section{Transient transfection and CAT assays}

Transfection was carried out by electroporation as described [35]. CAT activity was determined by two-phase diffusion assays [13] using $100 \mu \mathrm{g}$ of trophozoite extracts, $200 \mu \mathrm{l}$ of $1.25 \mathrm{mM}$ chloramphenicol and [ $\left.{ }^{14} \mathrm{C}\right]$-butyryl CoA (NEN Life Science Products). The background activity displayed by trophozoites transfected with the promoter-less vector was subtracted from activity obtained from cells transfected with each construct. Activities were expressed as relative activity with respect to that obtained from trophozoites transfected with the pA5'A3'CAT plasmid. CAT activity for each construct was assayed at least three times by duplicate. Data are expressed as mean of three independent experiments \pm S.D. for each group. Statistical significance was determined by Student's $t$-test and a difference of $\mathrm{p}<0.05$ was considered significant.

To determine the effect of heat shock on promoter activity, trophozoites transfected with the construct containing $683 \mathrm{bp}$ of the $E$ hrabB gene promoter were incubated at $37^{\circ} \mathrm{C}$ for $48 \mathrm{~h}$, and then at $42^{\circ} \mathrm{C}$ during 2 and $4 \mathrm{~h}$ before performing the CAT assays. 
Table I: Oligonucleotides used for amplify different fragments of the EhrabB promoter region.

\begin{tabular}{|c|c|c|}
\hline Name & Position ${ }^{a}$ & Sequence $^{b}$ \\
\hline Rab39-S & -39 & $\begin{array}{l}\left(5^{\prime}-\right. \\
\text { AAAACTGCAGAATAATAATTACAAAA } \\
\text { AG-3') }\end{array}$ \\
\hline Rabl47-S & -147 & $\begin{array}{l}\left(5^{\prime}-\right. \\
\text { AAAACTGCAGGTGTCTGTTAATTTCA } \\
\left.\text { AA- } 3^{\prime}\right)\end{array}$ \\
\hline Rab257-S & -257 & $\begin{array}{l}\left(5^{\prime}-\right. \\
\text { AAAACTGCAGGTTGAAAGGTTCTTAT- } \\
\left.3^{\prime}\right)\end{array}$ \\
\hline Rab273-S & -273 & $\begin{array}{l}\left(5^{\prime}-\right. \\
\text { AAAACTGCAGACAATCGCTGTCATTT } \\
\left.\text { GT- } 3^{\prime}\right)\end{array}$ \\
\hline Rab32I-S & -321 & $\begin{array}{l}\left(5^{\prime}-\right. \\
\text { AAAACTGCAGGCAATGCTTTGAGTCT } \\
\text { TT-3') }\end{array}$ \\
\hline Rab369-S & -369 & $\begin{array}{l}\left(5^{\prime}-\right. \\
\text { AAAACTGCAGGTTTTGAACTGAATAA } \\
\text { AC-3') }\end{array}$ \\
\hline Rab4I5-S & -415 & $\begin{array}{l}\left(5^{\prime}-\right. \\
\text { AAAACTGCAGGAACCTAAACTGTTCT } \\
\text { TC-3') }\end{array}$ \\
\hline Rab428-S & -428 & $\begin{array}{l}\left(5^{\prime}-\right. \\
\text { AAAACTGCAGTCTTAAATATTTGGAA } \\
\text { CC-3') }\end{array}$ \\
\hline Rab49I-S & -491 & $\begin{array}{l}\left(5^{\prime}-\right. \\
\text { AAAACTGCAGCATGAAACGCATCAGA } \\
\text { AG-3') }\end{array}$ \\
\hline Rab555-S & -555 & $\begin{array}{l}\left(5^{\prime}-\right. \\
\text { AAAACTGCAGGGAATAGCCATTCCCT } \\
\text { TA-3') }\end{array}$ \\
\hline Rab683-S & -683 & $\begin{array}{l}\left(5^{\prime}-\right. \\
\text { AAAACTGCAGACTCCCCACAAAAAGC } \\
\text { AT-3') }\end{array}$ \\
\hline $\begin{array}{l}\text { Rab- } \\
\text { AS33 }\end{array}$ & 24 & $\begin{array}{l}\left(5^{\prime}-\right. \\
\text { CCCAAGCTTCTTTAATGATGACACGT } \\
\text { СTCCCAT-3') }\end{array}$ \\
\hline
\end{tabular}

a Position with respect to first initiation transcription site.

b Underlined sequences correspond to the Pstl or Hindlll sites used to clone amplified fragments into the PBSCAT-ACT vector.

\section{Electrophoretic mobility shift assays (EMSA)}

DNA fragments of the EhrabB promoter gene (from -491 to -428 and from -428 to -369 ) were amplified and labeled by PCR using [ $\left.\alpha{ }^{32} \mathrm{P}\right] \mathrm{dATP}$. Reactions were carried out as described using specific oligonucleotides (Table 1) and the Pfx DNA polymerase (Invitrogen). Labeled fragments were separated on $12 \%$ nondenaturing polyacrylamide gels and purified. For other assays, a doublestranded oligonucleotide corresponding to position -428 to -412 (5'-TCTTAAATATTTGGAAC-3') was labeled at its 5 ' ends with T4 polynucleotide kinase (Invitrogen) and $[\gamma-$ 32P]ATP following standard procedures [48].

For electrophoretic mobility shift assays (EMSA), the [32P]-labeled fragments $(0.5-1 \mathrm{ng})$ were incubated at $4^{\circ} \mathrm{C}$ for $10 \mathrm{~min}$ with $30 \mu \mathrm{g}$ of nuclear extracts, $1 \mu \mathrm{g}$ of poly [d(I-
C)] (Amersham Biosciences) and 10\% glycerol in $12 \mathrm{mM}$ HEPES, pH 7.9, $60 \mathrm{mM} \mathrm{KCl,} 1 \mathrm{mM}$ DTT, 1 mM EDTA, 4 $\mathrm{mM}$ Tris-HCl, pH7.9, $1 \mathrm{mM}$ spermidine, $1 \mathrm{mM} \mathrm{MgCl}_{2}$. Competition assays were performed using as specific competitors a 150-fold excess of the same unlabeled fragment or a 150-fold excess of a double-stranded oligonucleotide containing the URE1 motif from the $h g l 5$ gene promoter [14], and as nonspecific competitors a 150-fold excess of a double-stranded oligonucleotide with similar base composition to URE1, but mingled sequence (5'-TTTTTCAGATAACAT-3') or $1.5 \mu \mathrm{g}$ of poly [d(I-C)] (350-fold excess). DNA-protein complexes were separated on $6 \%$ non-denaturing polyacrylamide gels and visualized with a phosphoimager analyzer (Bio-Rad).

\section{Abbreviations}

CAT: chloramphenicol acetyl transferase; EMSA: electrophoretic mobility shift assay; HSE: heat shock elements; URE1: upstream regulatory element 1; 5'UTR: 5' untranslated region.

\section{Competing interests}

The author(s) declares that there are no competing interests.

\section{Authors' contributions}

MRD carried out most of experiments and co-wrote the manuscript. ILP performed the primer extension assays. MBM carried out the real-time RT-PCR assays. CG participated in the study design and interpretation of data, EO participated in the study design, interpretation of data and co-wrote the manuscript. MAR conceived the project, supervised the experiments and co-wrote the manuscript. All authors read and approved the final manuscript.

\section{Acknowledgements}

This work was supported by the European Community and CONACyT (Mexico). We thank Dr. Jose de Jesus Trejo-Olivares for his support in realtime PCR assays. We thank Alfredo Padilla-Barberi for his invaluable technical assistance.

\section{References}

I. W.H.O.: Amoebiasis. WHO weekly epidemiol record 1997 , 72:97.- 100

2. Orozco E, de la Cruz Hernandez F, Rodriguez MA: Isolation and characterization of Entamoeba histolytica mutants resistant to emetine. Mol Biochem Parasitol 1985, I 5(I):49-59.

3. Rodriguez MA, Vargas MA, De Menezes LF, Lazard D, Orozco E: Phenotypic variation in clones of Entamoeba histolytica. Acta Parasitologica Turcica 1996, 20:203-210.

4. Orozco-Orozco ME, Martinez-Palomo A, Guarneros G: [Virulence and surface properties of various axenic strains of Entamoeba histolytica]. Arch Invest Med (Mex) 1980, II(I Suppl): $153-157$.

5. Bracha R, Mirelman D: Virulence of Entamoeba histolytica trophozoites. Effects of bacteria, microaerobic conditions, and metronidazole. J Exp Med 1984, I60(2):353-368.

6. Mirelman D, Bracha R, Chayen A, Aust-Kettis A, Diamond LS: Entamoeba histolytica: effect of growth conditions and bacterial associates on isoenzyme patterns and virulence. Exp Parasitol 1986, 62(I): $142-148$. 
7. De Menezes LF, Rodriguez MA, Vargas MA, Salgado LM, Orozco E: Effect of bacterial association on the phenotype and genotype of an Entamoeba histolytica clonal population. Invasion Metastasis 1997, 17(4): 176-188.

8. Meerovitch E, Ghadirian E: Restoration of virulence of axenically cultivated Entamoeba histolytica by cholesterol. Can J Microbiol 1978, 24(I):63-65.

9. Bruchhaus I, Roeder T, Lotter H, Schwerdtfeger M, Tannich E: Differential gene expression in Entamoeba histolytica isolated from amoebic liver abscess. Mol Microbiol 2002 44(4): $1063-1072$.

10. Gilchrist CA, Houpt E, Trapaidze N, Fei Z, Crasta O, Asgharpour A, Evans C, Martino-Catt S, Baba DJ, Stroup S, Hamano S, Ehrenkaufer G, Okada M, Singh U, Nozaki T, Mann BJ, Petri WA Jr.: Impact of intestinal colonization and invasion on the Entamoeba histolytica transcriptome. Mol Biochem Parasitol 2006.

II. Debnath A, Akbar MA, Mazumder A, Kumar S, Das P: Entamoeba histolytica: Characterization of human collagen type I and $\mathrm{Ca}+$ activated differentially expressed genes. Exp Parasitol 2005, II 0(3):214-219.

12. Leon-Avila G, Hernandez M, Camacho-Nuez M, Luna-Arias JP, Salazar I, Perez A, Munoz Mde L: Entamoeba histolytica up-regulates the Cdc48-like protein, an AAA family member, during the activation of trophozoites with collagen type I and calcium. Mol Biochem Parasitol 2006, I46(I): I I3-II9.

13. Buss H, Lioutas C, Dobinsky S, Nickel R, Tannich E: Analysis of the I 70-kDa lectin gene promoter of Entamoeba histolytica. Mol Biochem Parasitol 1995, 72( I-2): I- I0.

14. Purdy JE, Pho LT, Mann BJ, Petri WA Jr.: Upstream regulatory elements controlling expression of the Entamoeba histolytica lectin. Mol Biochem Parasitol I996, 78( I-2):9I-I03.

15. Gomez C, Perez DG, Lopez-Bayghen E, Orozco E: Transcriptional analysis of the EhPgpI promoter of Entamoeba histolytica multidrug-resistant mutant. I Biol Chem 1998 273(13):7277-7284

16. Perez DG, Gomez C, Lopez-Bayghen E, Tannich E, Orozco E: Transcriptional analysis of the EhPgp5 promoter of Entamoeba histolytica multidrug-resistant mutant. J Biol Chem 1998, 273(13):7285-7292

17. Ramirez ME, Perez DG, Nader E, Gomez C: Entamoeba histolytica: functional characterization of the -234 to -196 bp promoter region of the multidrug resistance EhPgpl gene. Exp Parasitol 2005, I I 0(3):238-243.

18. Marchat LA, Gomez C, Perez DG, Paz F, Mendoza L, Orozco E: Two CCAAT/enhancer binding protein sites are cis-activator elements of the Entamoeba histolytica EhPgp I (mdr-like) gene expression. Cell Microbiol 2002, 4(I I ):725-737.

19. Nieto A, Guillermo Perez D, Orozco E, Paz F, Gomez C: Entamoeba histolytica EhPgp5 transcriptional activation depends on putative emetine response elements. Exp Parasitol 2005, I I 0(3):233-237.

20. Gonzalez L Jr., Scheller RH: Regulation of membrane trafficking: structural insights from a Rab/effector complex. Cell 1999, 96(6):755-758.

21. Rodriguez MA, Garcia-Perez RM, Garcia-Rivera G, Lopez-Reyes I, Mendoza L, Ortiz-Navarrete V, Orozco E: An Entamoeba histolytica rab-like encoding gene and protein: function and cellular location. Mol Biochem Parasitol 2000, I 08(2): 199-206.

22. Guzman-Medrano R, Castillo-Juarez BA, Garcia-Perez RM, SalasCasas A, Orozco E, Rodriguez MA: Entamoeba histolytica: alterations in EhRabB protein in a phagocytosis deficient mutant correlate with the Entamoeba dispar RabB sequence. Exp Parasitol 2005, I I 0(3):259-264.

23. Marion S, Laurent C, Guillen N: Signalization and cytoskeleton activity through myosin IB during the early steps of phagocytosis in Entamoeba histolytica: a proteomic approach. Cell Microbiol 2005, 7(10): |504-15|8.

24. Okada M, Huston CD, Mann BJ, Petri WA Jr., Kita K, Nozaki T: Proteomic analysis of phagocytosis in the enteric protozoan parasite Entamoeba histolytica. Eukaryot Cell 2005, 4(4):827-83I.

25. Okada M, Huston CD, Oue M, Mann BJ, Petri WA Jr., Kita K, Nozaki $T$ : Kinetics and strain variation of phagosome proteins of Entamoeba histolytica by proteomic analysis. Mol Biochem Parasitol 2006, 145(2): 171-183.
26. Orozco E, Guarneros G, Martinez-Palomo A, Sanchez T: Entamoeba histolytica. Phagocytosis as a virulence factor. J Exp Med 1983, I58(5): I5II-I52I.

27. Garcia-Rivera G, Rodriguez MA, Ocadiz R, Martinez-Lopez MC Arroyo R, Gonzalez-Robles A, Orozco E: Entamoeba histolytica : a novel cysteine protease and an adhesin form the I/ $2 \mathrm{kDa}$ surface protein. Mol Microbiol 1999, 33(3):556-568.

28. Flores-Soto E, Azuara-Liceaga E, Lopez-Camarillo C, Orozco E: The Entamoeba histolytica Ehcp I 2 gene has a distal and weak promoter. Exp Parasitol 2005, I I 0(3):286-29|.

29. Azuara-Liceaga E, Flores-Soto E, Lopez-Camarillo C, Orozco E: Entamoeba histolytica: structural and functional analysis of the EhadhII2 gene promoter. Exp Parasitol 2005, I I 0(3):280-285.

30. Azizkhan JC, Jensen DE, Pierce AJ, Wade M: Transcription from TATA-less promoters: dihydrofolate reductase as a model. Critical reviews in eukaryotic gene expression 1993, 3(4):229-254.

3I. Bruchhaus I, Leippe M, Lioutas C, Tannich E: Unusual gene organization in the protozoan parasite Entamoeba histolytica. DNA Cell Biol 1993, I 2(10):925-933.

32. Gangopadhyay SS, Ray SS, Sinha P, Lohia A: Unusual genome organisation in Entamoeba histolytica leads to two overlapping transcripts. Mol Biochem Parasitol 1997, 89(I):73-83.

33. Luna-Arias JP, Hernandez-Rivas R, de Dios-Bravo G, Garcia J, Mendoza L, Orozco E: The TATA-box binding protein of Entamoeba histolytica: cloning of the gene and location of the protein by immunofluorescence and confocal microscopy. Microbiology I999, I45 ( Pt I):33-40.

34. Pickering BM, Willis AE: The implications of structured 5 untranslated regions on translation and disease. Semin Cell Dev Biol 2005, I6(I):39-47.

35. Nickel R, Tannich E: Transfection and transient expression of chloramphenicol acetyltransferase gene in the protozoan parasite Entamoeba histolytica. Proc Natl Acad Sci U S A 1994, 9I(15):7095-7098

36. Wu C: Heat shock transcription factors: structure and regulation. Annu Rev Cell Dev Biol 1995, I I:44 I-469.

37. Weber C, Guigon G, Bouchier C, Frangeul L, Moreira S, Sismeiro O, Gouyette C, Mirelman D, Coppee JY, Guillen N: Stress by heat shock induces massive down regulation of genes and allows differential allelic expression of the Gal/GalNAc lectin in Entamoeba histolytica. Eukaryot Cell 2006, 5(5):87I-875.

38. Mazel A, Leshem Y, Tiwari BS, Levine A: Induction of salt and osmotic stress tolerance by overexpression of an intracellular vesicle trafficking protein AtRab7 (AtRabG3e). Plant physiology 2004, I 34(I): I 18-128

39. Saloheimo $M$, Wang $H$, Valkonen $M$, Vasara $T$, Huuskonen $A$, Riikonen M, Pakula T, Ward M, Penttila M: Characterization of secretory genes yptl/yptA and nsfl/nsfA from two filamentous fungi: induction of secretory pathway genes of Trichoderma reesei under secretion stress conditions. Applied and environmental microbiology 2004, 70(I):459-467.

40. Brosche M, Fant C, Bergkvist SW, Strid H, Svensk A, Olsson O, Strid A: Molecular markers for UV-B stress in plants: alteration of the expression of four classes of genes in Pisum sativum and the formation of high molecular mass RNA adducts. Biochim Biophys Acta 1999, I 447(2-3): I85-198.

4I. Yamamoto A, Mizukami Y, Sakurai H: Identification of a novel class of target genes and a novel type of binding sequence of heat shock transcription factor in Saccharomyces cerevisiae. | Biol Chem 2005, 280( I 2): | | 9 |1-I| 1919.

42. Diamond LS, Harlow DR, Cunnick CC: A new medium for the axenic cultivation of Entamoeba histolytica and other Entamoeba. Trans R Soc Trop Med Hyg 1978, 72(4):43 I-432.

43. Entamoeba histolytica genome project [http://www.tigr.org/ $\mathrm{tdb} / \mathrm{e} 2 \mathrm{kl} / \mathrm{ehal} / \mathrm{s}$

44. TRANSFAC database [http://www.gene-regulation.com/cgi-bin/ pub/databases/transfac/search.cgi].

45. LALIGN server [http://www.ch.embnet.org/software/ LALIGN form.html].

46. Qvarnstrom Y, James C, Xayavong M, Holloway BP, Visvesvara GS, Sriram R, da Silva AJ: Comparison of real-time PCR protocols for differential laboratory diagnosis of amebiasis. Journal of clinical microbiology 2005, 43(I I):549|-5497. 
47. Livak KJ, Schmittgen TD: Analysis of relative gene expression data using real-time quantitative PCR and the 2(-Delta Delta C(T)) Method. Methods (San Diego, Calif 200I, 25(4):402-408.

48. Sambrook J, Russell DW: Molecular cloning. A laboratory manual. 3th edition. New York, Cold Spring Harbor Laboratory Press; 2001 .

Publish with Bio Med Central and every scientist can read your work free of charge

"BioMed Central will be the most significant development for disseminating the results of biomedical research in our lifetime. " Sir Paul Nurse, Cancer Research UK

Your research papers will be:

- available free of charge to the entire biomedical community

- peer reviewed and published immediately upon acceptance

- cited in PubMed and archived on PubMed Central

- yours - you keep the copyright

Submit your manuscript here:

http://www.biomedcentral.com/info/publishing_adv.asp
BioMedcentral 